\title{
De-noising Method in the Wavelet Packets Domain for Phase Images
}

\author{
Juan V. Lorenzo-Ginori and Héctor Cruz-Enriquez \\ Center for Studies on Electronics and Information Technologies, Universidad Central \\ "Marta Abreu" de Las Villas, Carretera a Camajuaní, km 5 1⁄2, \\ Santa Clara, VC, CP 54830, Cuba \\ juanl@uclv.edu.cu, hcruz@uclv.edu.cu \\ http://www.fie.uclv.edu.cu
}

\begin{abstract}
Complex images contaminated by noise appear in various applications. To improve these phase images, noise effects, as loss of contrast and phase residues that deteriorate the phase unwrapping process, should be reduced. Noise reduction in complex images has been addressed by various methods, most of them dealing only with the magnitude image. Few works have been devoted to phase image de-noising, despite the existence of important applications like Interferometric Synthetic Aperture Radar (IFSAR), Current Density Imaging (CDI) and Magnetic Resonance Imaging (MRI). In this work, several de-noising algorithms in the wavelet packets domain were applied to complex images to recover the phase information. These filtering algorithms were applied to simulated images contaminated by three different noise models, including mixtures of Gaussian and Impulsive noise. Significant improvements in SNR for low initial values $(\mathrm{SNR}<5 \mathrm{~dB}$ ) were achieved by using the proposed filters, in comparison to other methods reported in the literature.
\end{abstract}

\section{Introduction}

Images produced by systems such as Synthetic Aperture Radars (IFSAR), Current Density Imaging (CDI) and Magnetic Resonance Imaging (MRI) appear as arrays of complex numbers and are affected by the presence of noise. These images suffer in many cases from a poor signal to noise ratio (SNR).

Complex images allow the use of both magnitude and phase information, depending on the type of application considered. The presence of noise in the complex images can be originated by numerous causes as can be the noise produced by the acquisition hardware, physiological noise originated from the patients, noisy artifacts provoked by movements during image acquisition (MRI, CDI) and the presence of phase jitter that can appear during the acquisition of signals obtained by IFSAR. In all the previously mentioned cases noise not only produces a deterioration in SNR and loss of contrast in the image, but also it introduces phase residues that will affect negatively the later phase unwrapping process, that is unavoidable in most applications where the analysis is based upon the phase information from the complex image obtained. 
In this work three noise models were considered. These are combinations of additive white Gaussian (AWGN) and impulsive noise in various proportions. Preliminary results obtained in [1] have been considered as a reference for comparison. The algorithms developed in [2, 3] for magnitude images have been also implemented for comparison for phase images, in order to better show the effectiveness of the filtering methods introduced here. A description of the noise models associated to complex images have been discussed in [2, 3]. Most de-noising algorithms developed for complex images have assumed zero-mean AWGN, which contaminate independently the real and imaginary parts of the complex image. Noise distribution in the magnitude image is usually assumed to have a zero-mean Rician distribution, which behaves as a Gaussian distribution for high SNR and as a Rayleigh one for low SNR. Our main interest is centered in the phase images in low SNR environments.

In a previous work [2], a de-noising algorithm was reported based in a Wiener filter that reduces noise in a very effective way in the magnitude image and it is claimed that it can also make this simultaneously in the phase image. Another approach based in nonlinear filtering was introduced in [1], and de-noising methods using the Wavelet Transform were introduced and tested in [7]. In this work we pursue to show some considerations related to wavelet packets de-noising for phase images that differ in a certain extent from its application to magnitude images and that exhibited some advantage in SNR improvement when compared to the previous cited works.

\section{Materials and Methods}

\subsection{Simulated Image}

The complex simulated image was built in similar way as in previous works $[1,2]$. This consists in a magnitude image formed as a 64 x 64 pixels square with intensity 210 (bright region) which is centered inside another square of size 128 x 128 with 90 units intensity (dark region). The original unwrapped phase image was defined as the bi-dimensional Gaussian function

$$
\varphi_{u v}=A \exp \left(\frac{(u-64)^{2}}{\sigma_{u}^{2}}+\frac{(v-64)^{2}}{\sigma_{v}^{2}}\right),
$$

where $u$ and $v$ are the variables associated to the coordinate axes. For the rest of the variables in equation (1), the following values were used: $A=7 \pi, \sigma_{u}^{2}=3500$ and $\sigma_{v}^{2}=1000$

The complex image was formed from the magnitude and wrapped phase images. It was contaminated with various proportions of AWGN and impulsive noise, independently for the real and imaginary parts. The various noise models employed here are shown in Table 1. This table shows the standard deviation values for a 
Table 1. Noise models

\begin{tabular}{|c|c|c|}
\hline Noise model & $\sigma$ & $P_{I}, \%$ \\
\hline 1 & 60 & 0 \\
\hline 2 & 70 & 3 \\
\hline 3 & 90 & 5 \\
\hline
\end{tabular}

Gaussian probability density function with zero mean and the corresponding percentages of impulsive noise.

The impulsive noise was modeled in the same way as in [1], where the probability of occurrence of an impulse for any part, real or imaginary, is given by

$$
p=1-\sqrt{1-P_{I}}
$$

In Table 1 are shown the global percentages $P_{I}$ of the impulses to be generated. The $p$ value is to be divided evenly for the contribution of positive and negative pulses. Both the image and the noise were modeled considering an 8-bit resolution for the representation of their numerical values.

\subsection{Measurement Parameters}

In order to demonstrate the effectiveness of the algorithms and compare them with previous works reported in the literature we performed a set of measurements similar to those performed in [1], where we determined the values of SNR, the number of phase residues (RES), the standard deviation (STDV) and the normalized mean square error (NMSE), defined as

$$
N M S E=\frac{\sum_{i} \sum_{j}\|\varphi(i, j)-\hat{\varphi}(i, j)\|^{2}}{\sum_{i} \sum_{j}\|\varphi(i, j)\|^{2}}
$$

where $\varphi$ is the original unwrapped phase, $\hat{\varphi}$ is the recovered unwrapped phase after filtering and $(i, j)$ are the pixel values in the direction $(u, v)$.

SNR was calculated as

$$
S N R=10 \log _{10}\left(\frac{1}{N M S E}\right)
$$

The amount of phase residues that appear both in the noisy and in the de-noised signals were calculated by applying systematically the expression

$$
\varphi(r)=\oint_{C} \nabla \varphi(r) \cdot d r=2 K \pi
$$


Here $\varphi(r)$ is the signal phase, $\nabla \varphi(r)$ is the phase gradient and $K$ is an integer number that accounts for the phase residues enclosed by the contour $C$.

\subsection{De-noising Algorithms}

Two new algorithms in the wavelet packets domain were proposed here to increase SNR in phase images. These filtering processes begin with the application of the bidimensional Discrete Wavelet Packet Transform (DWPT-2D) to both the real and imaginary parts of the noisy complex image $z_{n}$. From this transformation, the noisy DWPT-2D complex coefficients $\boldsymbol{c}_{\boldsymbol{j}, \boldsymbol{o}}^{\boldsymbol{c h}}$ were obtained, where the index $c h$ indicates whether the coefficient belongs to the real ( $r e$ ) or imaginary ( $\mathrm{im}$ ) parts of the complex image, and the terms $j$ and $o$ indicate the decomposition level and the orientation (horizontal, vertical or diagonal), respectively.

The expression of the transformation $T$ for the DWPT-2D is given by

$$
c_{j, o}^{c h}=T_{D W P T-2 D}\left[z_{n}\right] .
$$

After calculating this transformation, the wavelet packet coefficients are appropriately thresholded obtaining $\left\{\widehat{\boldsymbol{c}}_{\boldsymbol{j}, \boldsymbol{o}}^{\boldsymbol{c h}}\right\}$, and the synthesis equation associated to the DWPT equation (6) was applied later, resulting in

$$
\widehat{z}=T_{D W P T-2 D}{ }^{-1}\left[\widehat{c}_{j, o}^{c h}\right] .
$$

The first filtering method described is based in the classical soft thresholding of the wavelet packet coefficients (called SOFT_WP here). Thresholding was applied independently to the real and imaginary parts, as

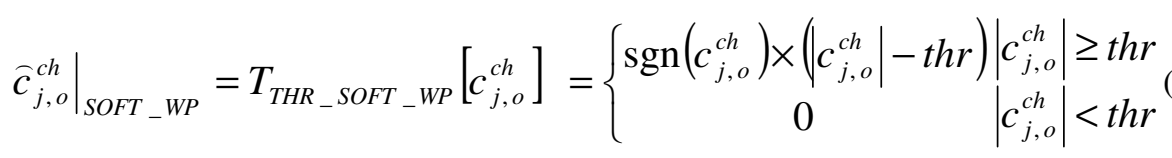

where $t h r$ is the threshold value, whose calculation will be discussed in paragraph 2.4.

The second filtering method (called A_SOFT_WP), thresohlding was applied to the magnitude wavelet packet coefficients, instead of doing this for the real and imaginary parts independently where

$$
\left|c_{j, o}\right|=\sqrt{\left(c_{j, o}^{R E}\right)^{2}+\left(c_{j, o}^{I M}\right)^{2}} .
$$


The filtering transformation was in this case

$$
\begin{gathered}
\mid \widehat{c}_{j, o} \|_{A_{-} S O F T_{-} W P}=T_{T_{H R_{-} S O F T_{-} W P}}\left[\left|c_{j, o}\right|\right]= \\
\begin{cases}\operatorname{sgn}\left(\left|c_{j, o}\right|\right) \times\left(\left|c_{j, o}\right|-t h r\right), & \left|c_{j, o}\right| \geq t h r_{G} \\
0, & \left|c_{j, o}\right|<t h r_{G}\end{cases}
\end{gathered}
$$

where the threshold is obtained from the thresholds for the real and imaginary parts as

$$
t h r_{G}=\left[\left(t h r_{R E}\right)^{2}+\left(t h r_{I M}\right)^{2}\right]^{\frac{1}{2}}
$$

Various other filtering alternatives were devised and tested in the wavelet packet domain, from which we have illustrated here only the most representative cases with which we obtained the best results.

\subsection{Threshold Calculation}

There exist several methods to obtain the noise standard deviation from noisy data and from this to obtain the threshold values to be used [2, 3]. In most applications noise can be considered uncorrelated and independent from the decomposition level, frequency and orientation. Having this in mind, the best alternative for noise estimation was to apply the DWPT-2D at the finest scale, e. g. the first decomposition level. This is the median absolute deviation (MAD) estimate used in [4], with which the resulting threshold is

$$
\operatorname{thr}=\operatorname{median}\left(\left|c_{1, o}^{c h}\right|\right) / 0.6745^{\circ}
$$

Here median $\left(\left|c_{1, o}^{c h}\right|\right)$ is the value of the statistical median of the array formed by the absolute value of the wavelet packets coefficients from the first decomposition level. The global threshold for de-noising is obtained by a wavelet packet coefficients selection rule using a penalization method provided by Birge-Massart [6].

\section{Results}

Performance evaluation for the filters described above was realized using a simulated complex image as it was described in 2.2. Tables 2 and 3 show the results obtained for the two filters described above, corresponding to SNR and NMSE for two out of the three noise models shown in Table 1. In both filters the wavelet packet Bior 2.6 
was employed, with a number $J=4$ of decomposition levels, which led to good results. As can be observed, in all cases the noisy phase image had SNR values less than $5 \mathrm{~dB}$. This was determined basically because our objective was to improve phase images in low SNR environments. Figure 1 shows in the first column the original (wrapped and unwrapped) simulated images, in the second column the contaminated images (wrapped and unwrapped with an algorithm that does not tolerate phase residues) and in the third column the results obtained once the de-noising process was applied with the filter SOFT_WP. The simulated complex image in this experiment was contaminated with noise model $2(\mathrm{stdv}=70$ and $\mathrm{imp}=3 \%)$.

Figure 2 shows a comparison between the algorithm proposed here (SOFT_WP) and the best of the previous algorithms published in the literature (A_H_S_U) [7]. In this case the simulated complex image was contaminated with noise model 3 (stdv = 90 and imp $=5 \%$ ) with the objective of illustrating the effectiveness of the use of wavelet packets in situations of very low signal to noise. The unwrapped phase image shows clearly the improvement obtained with the SOFT_WP filter versus the A_H_S_U filter.

Table 2. Results of filtering in terms of NMSE and SNR, noise models 2 and 3

\begin{tabular}{|c|c|c|c|c|c|c|}
\hline \multirow[t]{3}{*}{ Image: } & Wave & et: Bior2.6 & \multicolumn{2}{|c|}{ Noiseless } & \multirow[t]{2}{*}{$0 \mathrm{~T}$} & \multirow[t]{2}{*}{ rials: 2} \\
\hline & & & Noise & model & & \\
\hline & & 2 & & & 3 & \\
\hline filter & NMSE & STDV & SNR & NMSE & STDV & SNR \\
\hline NONE & 0.9144 & $5.39 e-001$ & 0.97 & 1.2821 & $7.85 e-001$ & -0.61 \\
\hline A_SOFT_WP & 0.0006 & $4.81 e-004$ & 32.88 & 0.0068 & $7.17 e-003$ & 23.49 \\
\hline SOFT_WP & 0.0004 & $3.50 e-005$ & 34.11 & 0.0027 & $2.77 e-003$ & 27.50 \\
\hline
\end{tabular}

Table 3. Results of filtering in terms of phase residues, noise models 2 and 3 Image: Image 1 Wavelet: Bior2.6 Noiseless residues: 0 Trials: 20
Noise model

$\begin{array}{lrrrr}\text { Filter } & \text { Nres } & \text { stdv } & \text { Nres } & \text { stdv } \\ \text { NONE } & 1396.80 & 47.75 & 2149.70 & 44.17 \\ \text { A_SOFT_WP } & 0.20 & 0.62 & 4.10 & 2.55 \\ \text { SOFT_WP } & 0.00 & 0.00 & 2.10 & 2.00\end{array}$



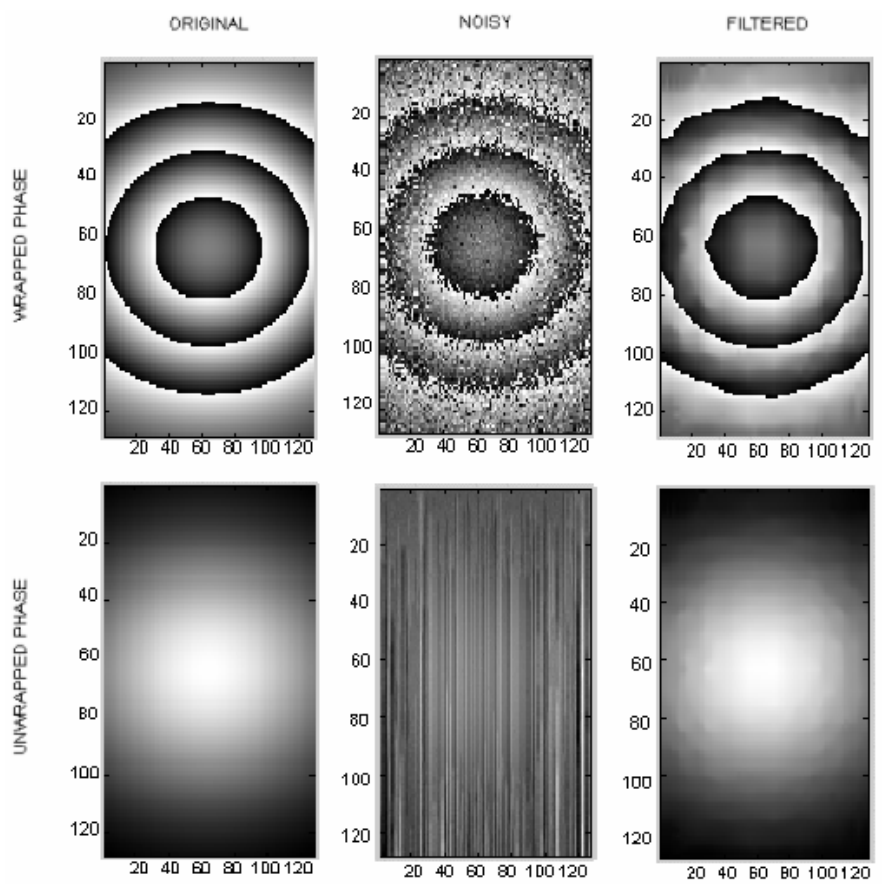

Fig. 1. De-noising of simulated image, wavelet packet Bior2.6, $J=4$, filter SOFT_WP, noise model 2
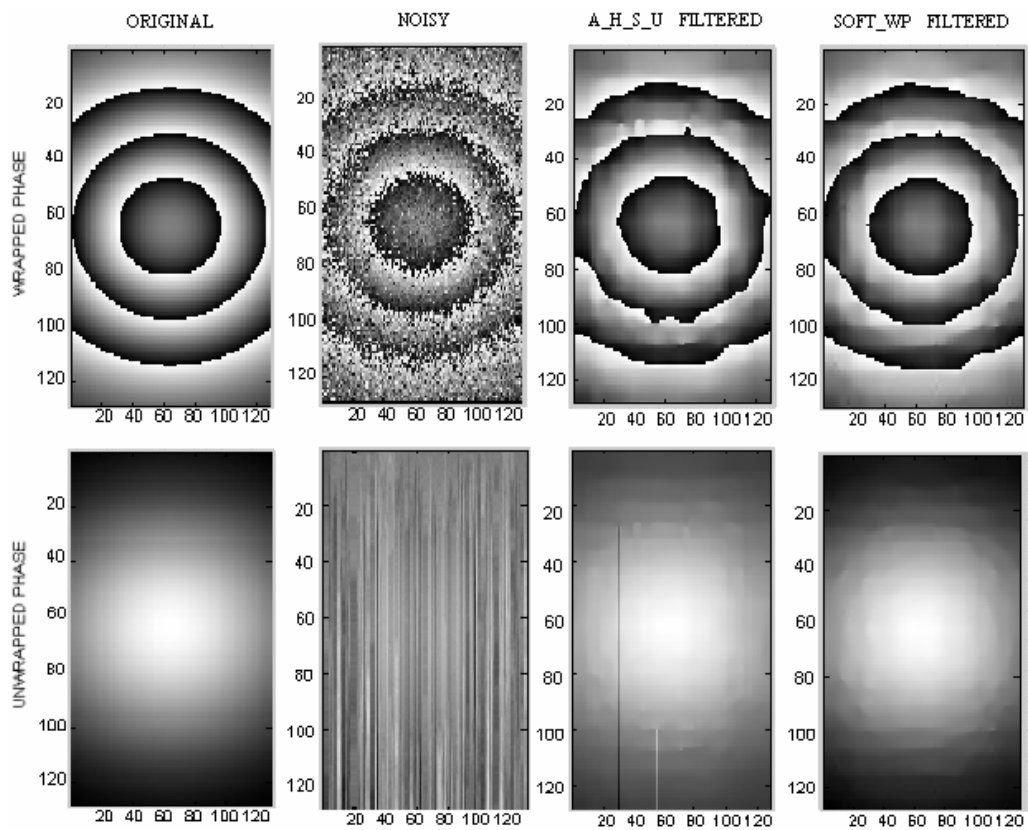

Fig. 2. De-noising of simulated image, wavelet packet Bior2.6, $J=4$, filters A_H_S_U and SOFT_WP, noise model 3 


\section{Discussion and Conclusions}

The proposed methods constitute a new alternative for phase image de-noising that differ from the traditional wavelet-domain methods [2, 3, 4, 5, 7] that are based in Wiener filtering or in soft thresholding and phase preservation of the wavelet coefficients in the wavelet domain.

The use of soft thresholding techniques reduced noise significantly, showing a high and stable SNR gain for all the noise models used in this work. The only drawback present in the wavelet based methods was the poor edge preservation in some regions of interest in the image. The methods based on wavelet packets showed a noticeable reduction of this negative effect.

Through the simulation experiments performed here, it was possible to conclude that it is the magnitude image, and not the phase one, the most sensitive to phase changes in the wavelet packets coefficients. This is because it was observed that the magnitude image was degraded when the real and imaginary parts of the wavelet packet coefficients were filtered independently, while this process led to an improvement of the phase image.

These results indicated a significant noise reduction, which surpass previous results reported in the literature $[1,7]$ and in this case without the need of an excessive computational burden.

\section{References}

1. Lorenzo-Ginori, J. V., Plataniotis, K. N. and Venetsanopoulos, A. N.: Non linear filtering for phase image de-noising.. IEE Proc.-Vis. Image Signal Process, Vol 49(5) 290-296, October 2002.

2. Alexander, M. E. , Baumgartner, R., Summers, A. R., Windischberger, C. , Klarhoefer, M., Moser, E. and Somorjai, R. L,: A Wavelet-based Method for Improving Signal-to-noise Ratio and Contrast in MR Images. Magnetic Resonance Imaging 18 (2000) 169-180.

3. R. D.Nowak: Wavelet-Based Rician Noise Removal for Magnetic Resonance Imaging. IEEE Transactions on Image Processing Vol. 8 (10) 1408-1419, 1999.

4. H.Braunisch, W. Bae-ian, and J. A.Kong,: Phase unwrapping of SAR interferograms after wavelet de-noising. In: IEEE Geoscience and Remote Sensing Symposium, IGARSS 2000. 2 (2000) $752-754$.

5. S. Zaroubi, and G. Goelman: Complex De-noising of MR Data Via Wavelet Analysis: Application to Functional MRI. Magnetic Resonance Imaging 18 (2000) 59-68.

6. M. Misiti et al, Wavelet Toolbox user's guide, The MathWorks Inc., Natick, MA, 2000.

7. H. Cruz-Enríquez and J. V. Lorenzo-Ginori, Wavelet-based methods for improving signalto-noise ratio in phase images, paper accepted in the International Congress on Image Analysis and Recognition ICIAR 2005, (to be published in LNCS), September 28-30, 2005, Toronto, Canada. 